\title{
A CONTRIBUTION TO THE MICROSCOPICAL ANATOMY OF THE SYMPATHIZING EYE
}

BY

\section{R. AfFleck GReeves}

IN comparison with the number of existing publications concerned with the microscopical anatomy of the exciting eye in sympathetic ophthalmitis, those dealing with the sympathizing eye are relatively few. In most ophthalmological text-books indeed, it is stated that the character of the histological changes found in exciting and sympathetic eyes is identical.

The subject of this communication is a number of sympathizing eyes which I have had the opportunity of examining after they had been sectioned. These are 13 in number, they have been collected from different sources, and their history, I think, leaves no doubt as to the true nature of their pathological condition; in all of them the original perforating injury to the exciting eye was an intraocular operation.

The order in which I have arranged this series of cases corresponds to the particular length of time which elapsed between the date on which inflammatory signs were first observed in the sympathizing eye and that on which. it was enucleated in each case. In Case 1 this period was the shortest, and in case 10 it was most prolonged. In the 3 cases which I have placed last on the list, the exact duration of this period, although undoubtedly a long one, is somewhat uncertain.

A study of the foregoing cases shows that the particular character of the inflammatory changes occurring in sympathizing eyes is not related to the duration of the disease, and that this statement also applies to the distribution of such changes in the various ocular tissues. Nor can any relationship be found between either of these factors and the length of time elapsing between the injury to the exciting eye and the onset of inflammation in the sympathizing eye.

In 7 cases the infiltrating inflammatory cells were purely lymphocytes and plasma cells, in 6 cases epithelioid cells were present and in 3 of the latter, giant cells appeared. The only constant factor common to all these cases is the presence of an irido-cyclitis which varied considerably in character and intensity. The choroid was found to be normal in 5 cases, while in 6 cases areas of infiltration were found consisting of lymphocytes and plasma cells only. Epithelioid cells occurred in the choroid. in 2 cases, and giant cells in one case only. These areas of choroidal 
infiltration consisted of localized patches, always situated in the outer layers of the choroid, the capillary layer being free except in one case, No. 13, in which the whole thickness of the choroid was involved. Also the posterior section of the choroid, that immediately surrounding the optic disc, was consistently the most densely infiltrated part. Both these findings are in accordance with the conditions usually found in exciting eyes and I regard them as salient characteristics of sympathetic disease.

The vitreous showed some inflammatory cells in most cases.

The optic nerve was normal in 5 cases and oedematous in 8 cases in which the sheaths of the central vessels showed lymphocytic infiltration.

The optic nerve sheath was infiltrated in one case only and the sheaths of the ciliary vessels in 4 cases.

An interesting point is the spontaneous rupture of the lens capsule which was found in 3 cases, in none of which had there been any operative interference. In 2 of these cases, in both of which the disorganized iris and swelling lens matter were jumbled together in a necrotic mass, the intra-ocular pressure was low, while in the third the tension was raised.

Organized plastic exudate in the anterior chamber was found in 3 cases in which no operative interference had been undertaken and its occurrence does not support Fuchs' view that plastic exudation in exciting eyes affected with sympathetic disease is due to secondary infection and not part of the specific process.

In none of the cases did such infiltration as was present in the iris show any particular tendency to be situated in the posterior layers, and no Fuchs-Dalen bodies were seen.

From a study of these 13 cases I think it is reasonable to draw the conclusion that no special characteristic of the sympathetic inflammatory process is invariably found in the sympathizing eye on microscopical examination.

Case 1. Male, age 60 years. Signs of sympathetic uveitis were observed 5 weeks after operation on the other eye. Enucleation was performed 3 months later because the eye became glaucomatous and painful.

Microscopical appearances. - Scattered nodular lymphocytic and plasma cell infiltration of the iris, with a few epithelioid cells in some of the nodules. Slight lymphocytic infiltration of the ciliary body. The anterior uveitis was not plastic in character. Nodular lymphocytic and plasma cell infiltrations were present in the outer layers of the choroid in the neighbourhood of the optic disc. No epithelioid or giant cells were seen. Retinal vessels, optic disc, vitreous and ciliary vessels and nerves were free from infiltration:

Case 2. Female, age unknown. Signs of sympathetic uveitis appeared 2 months after operation on the other eye and an iridectomy was performed for the relief of secondary glaucoma. The eye was enucleated $3 \frac{1}{2}$ months after the first appearance of inflammatory signs.

Microscopical appearances.-A layer of lymphocytes was present on the posterior corneal surface, and nodular lymphocytic infiltration of the iris and ciliary/ body; 


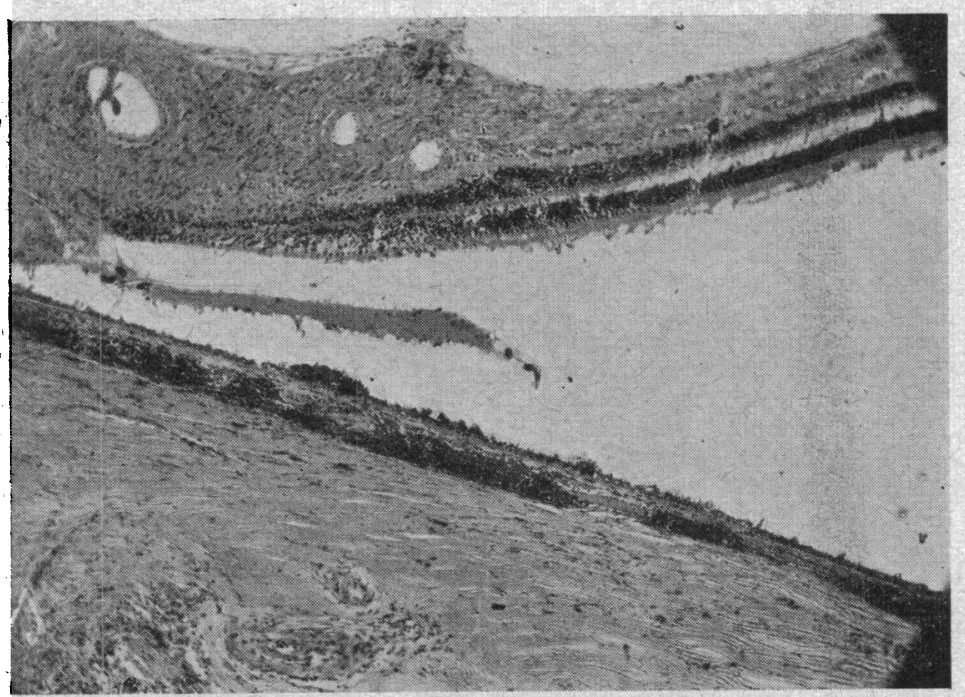

Fig. 1.

Photo by E. V. Willmot

Showing nodular lymphocytic infiltration in the posterior layers of the choroid in Case 1. The retinal detachment is an artefact.

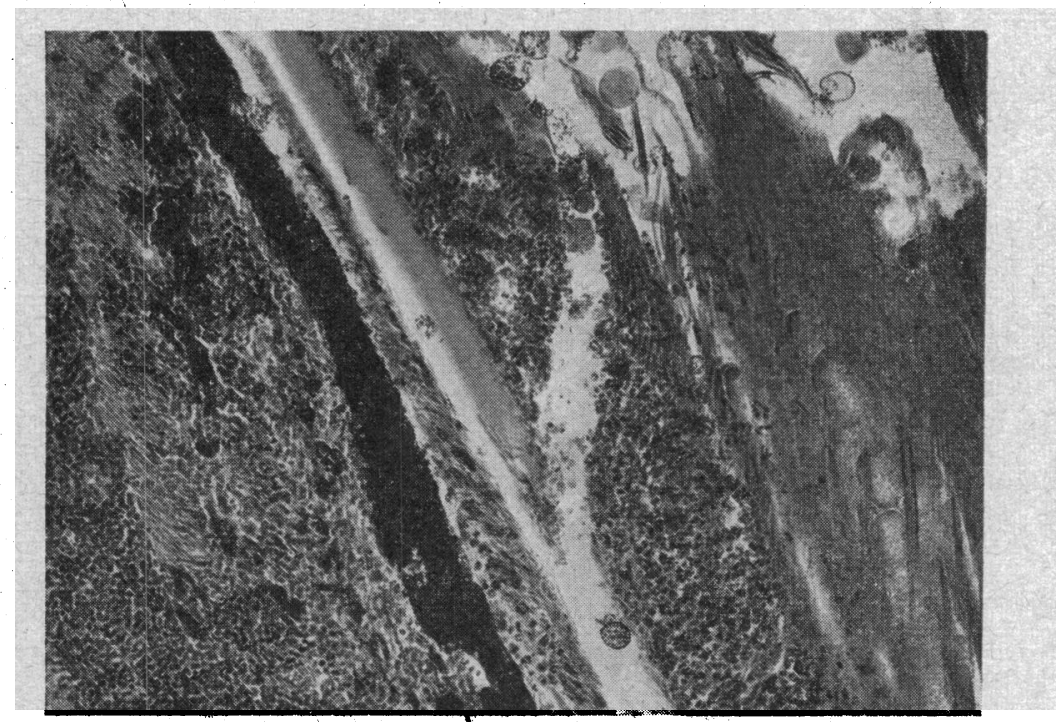

FIG. 2.

Photo by $E$. V. Willmott

Showing the ruptured lens capsule in Case 3, and the invasion of the lens substance by inflammatory cells. 
no epithelioid cells were seen. There is slight lymphocytic infiltration of the choroid near the optic disc. The central vessels and larger retinal vessels show marked lymphocytic infiltration in their sheaths. The ciliary vessels also show some lymphocytic infiltration. There is scattered lymphocytic infiltration in the vitreous.

Case 3. Female, age 49 years. Signs of sympathetic uveitis were observed 3 months after operation on the other eye, and 5 months later the eye was enucleated because of secondary glaucoma.

Microscopical appearances.-Dense coagulum was present in the anterior chamber and dense infiltration, mainly lymphocytic, in the iris, with a few epithelioid and giant cells in the denser nodules. There is much plastic exudation, with an organized membrane in the pupil. The ciliary body shows dense lymphocytic infiltration with a few epithelioid cells. Localized patches of plasma cells, lymphocytes, and a few epithelioid cells occur in the outer layers of the choroid near the optic disc. There is some lymphocytic infiltration round the central vessels. The ciliary vessels and nerves also show some perivascular infiltration. The optic nerve and its sheath are also invaded by lymphocytes The lens capsule is ruptured, with invasion of the lens substance by inflammatory cells.

Case 4. Male, age 60 years. Signs of sympathetic uveitis were observed 2 months after operation on the other eye. Enucleation took place 5 months later because of secondary glaucoma, for which a paracentesis had already been performed.

Microscopical appearances. Plastic exudate, partly organized, is present in the anterior chamber. Lymphocytic and plasma cell infiltration only is seen in the iris and ciliary body. The choroid and optic nerve are normal. The vitreous is slightly infiltrated anteriorly.

Case 5. Female, age 54 years. Signs of sympathetic uveitis were observed 3 months after operation on the other eye. 10 months later the eye was enucleated because of secondary glaucoma for which a paracentesis had already been done. Dense lymphocytic and plasma cell infiltration occur in the iris and ciliary body, and a similar type of nodular infiltration is seen in the outer layers of the posterior choroid. The optic disc shows infiltration with lymphocytes anteriorly and there is lymphocytic infiltration of the sheaths of the larger vessels. No epithelioid or giant cells are observed. No ciliary vessel or nerve infiltration is seen. The vitreous shows a few scattered inflammatory cells.

Case 6. Female, age 59 years. Sympathetic uveitis was obsęrved 1 year and 5 months after operation on the other eye, and 11 months later, when the eye was shrinking it was enucleated. The iris and ciliary body are mainly disorganized, and represented by a mass of granulation tissue containing lymphocytes, plasma cells, epithelioid and giant cells. The choroid shows localized areas of lymphocytic infiltration posteriorly, but no epithelioid or giant cells. The optic disc shows lymphocytic infiltration in the sheaths of the central vessels. The sheaths of the ciliary vessēls and nerves are also infiltrated. The vitreous is detached and infiltrated. The lens capsule is ruptured.

Case 7. Male, age 70 years. Sympathetic uveitis was first observed a year after operation on the other eye. Enucleation took place a year later because of secondary glaucoma.

Microscopical appearances.-Plastic exudation almost fills the anterior chamber, but some K.P. can be seen. The' iris and ciliary body show scattered infiltration, with lymphocytes and plasma cells. In the posterior part of the choroid are localized patches of lymphocytes and plasma cells, but no epithelioid cells. The optic disc, central vessels, and ciliary vessels and nerves are normal. A few inflammatory cells are seen in the vitreous.

Case 8. Female, age 64 years. Signs of sympathetic uveitis were observed 5 months after operation on the other eye. 1 year and 3 months later, the eye was enucleated because of secondary glaucoma, for which a paracentesis had already been performed.

Microscopical appearances.-Organized exudate is seen in the anterior chamber. The iris and ciliary body show nodular infiltrates with lymphocytes and plasma cells, but no epithelioid cells. The choroid is normal. The optic nerve is cupped 
and atrophic with a few lymphocytes in the sheaths of the larger vessels. The ciliary vessels, nerves and vitreous are normal.

Case 9. Male, age 70 years. Signs of sympathetic uveitis were observed 4 months after operation on the other eye. Enucleation took place 16 months later. Microscopical appearances.-Nodular infiltration of the ciliary body and iris, with lymphocytes and plasma cells, and epithelioid cells, but no giant cells are seen. The choroid, ciliary vessels and nerves are normal. There is some infiltration of the larger vessels on the optic disc and some inflammatory cells in the anterior vitreous.

Case 10. Age, sex, and other particulars unknown. Enucleation took place 18 months after operation on the other eye. The eye was then shrinking.

Microscopical appearances.-Coagulum was present in the anterior chamber. The iris and ciliary body were mainly disorganized and showed dense lymphocytic and plasma infiltration with some epithelioid cells. No giant cells were seen. The choroid and optic nerve were normal as well as the ciliary vessels. The lens capsule was ruptured.

Case 11. Female, age 50 years. The dates are uncertain, but the eye was excised over 4 years after the operation on the other because it was then blind and painful. The iris and ciliary body show nodular lymphocytic and plasma cell infiltration, with no epithelioid cells. The choroid is normal. The optic nerve and the sheaths of the larger vessels are infiltrated anteriorly. The ciliary vessels and nerves are free from infiltration. The vitreous shows many inflammatory cells.

Case 12. Female, age 67 years. The eye was enucleated 9 years after operation on the other eye, because it became blind and painful.

Microscopical appearances.-The iris and ciliary body are slightly infiltrated with lymphocytes and plasma cells, polymorphonuclears are visible in the anterior chamber. Some nodular lymphocytic infiltration occurs in the posterior part of the choroid in its outer layers. The vitreous is clear. The optic nerve was not seen.

Case 13. Female, age 55 years. A left iridectomy was performed. The' right

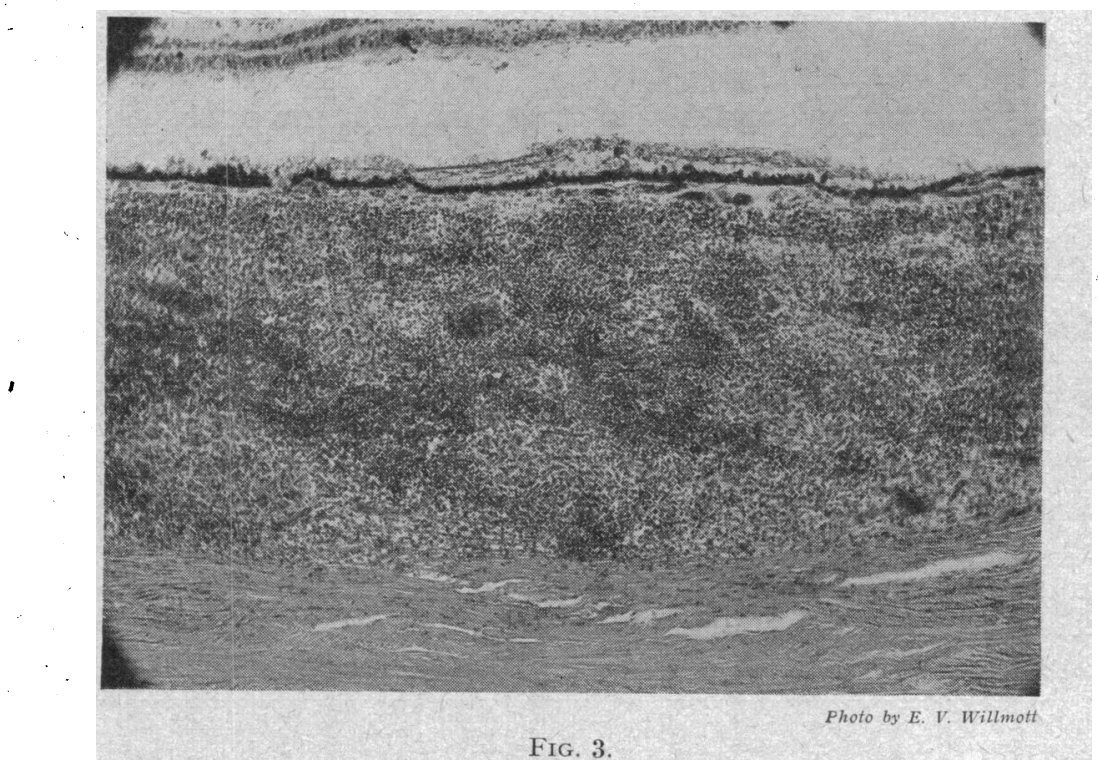

Section showing the choroid in Case 13. Dense infiltration with lymphocytes and epithelioid cells. The retinal detachment is an artefact. 
eye became painful and glaucomatous some weeks later and was trephined 4 months after the original operation on the left eye. It was enucleated a month later.

Microscopical Sections.-This eye is the only one of the series which shows all the characteristics recognized as peculiar to advanced sympathetic disease in an exciting eye. The whole of the uveal tract is a mass of granulation tissue in which all types of inflammatory cell are seen, and which shows nodules containing epithelioid and giant cells. The central vessels, optic disc, and the nerve sheath, as well as the ciliary vessels and nerves, are infiltrated with lymphocytes. The lens capsule is ruptured, but in this case the rupture may have occurred when the operation for the relief of secondary glaucoma was performed.

\title{
THE OFF/ON-RATIO OF THE ISOLATED ON-OFF- ELEMENTS IN THE MAMMALIAN EYE
}

\author{
BY \\ RAGNAR GRANIT \\ (FROM THE NOBEL INSTITUTE FOR \\ NEUROPHYSIOLOGY, STOCKHOLM)
}

IN this communication I would like to draw attention to some aspects of the micro-electrode work with the retina of the dark adapted decerebrate cat which have come to the fore after the appearance of my (very much delayed) summary in 1947. Some new results and concepts have emerged from the further analysis of several hundred isolated fibres in this retina and it is possible that there is something in this work that may interest the clinician also. It is not my intention to review experiments published elsewhere, except in so far as they are necessary for this discussion of concepts and principles.*

The retinal elements are either on-elements, off-elements or onoff-elements. The on-elements respond to light with a stream of impulses, the off-elements with a discharge at the cessation of illumination and the on-off-elements combine these two properties. For the discrimination of light and colours the on-off-elements' must be the most important ones. In the cat's eye they are also the most numerous ones; there Miss Tansley and I found 80 per cent. on-off-elements. The on-elements turned up in 16 per cent. of our total of 164 elements. We have reasons to believe them to be pure rod-elements. The on-off-elements may differ in colour sensitivity at "on " and "off." For this reason it is impossible to assume that the on and off-components are set up by identical

\footnotetext{
- The original papers will be found in the $J l$. Physiol., and $J l$. Neuro-Physiol., for 1947. The latest one is due to appear in the latter Journal within a few months. The summary referred to is " Sensory Mechanisms of the Retina," Oxford Univ. Press, 1947.
} 